\title{
A Middle-Range Theory of Self-Care of Chronic Illness
}

\author{
Barbara Riegel, Tiny Jaarsma and Anna Strömberg
}

\section{Linköping University Post Print}

\section{Tweet}

N.B.: When citing this work, cite the original article.

Original Publication:

Barbara Riegel, Tiny Jaarsma and Anna Strömberg, A Middle-Range Theory of Self-Care of Chronic Illness, 2012, Advances in Nursing Science, (35), 3, 194-204.

http://dx.doi.org/10.1097/ANS.0b013e318261b1ba

Copyright: Lippincott, Williams \& Wilkins

http://www.lww.com/

Postprint available at: Linköping University Electronic Press

http://urn.kb.se/resolve?urn=urn:nbn:se:liu:diva-81836 


\title{
A Middle-Range Theory of Self-Care of Chronic Illness
}

\author{
Barbara Riegel, DNSc, RN, FAAN, FAHA \\ Professor and Edith Clemmer Steinbright Chair of Gerontology, \\ University of Pennsylvania School of Nursing \\ Philadelphia, PA, USA
}

Division of Nursing Science, Department of Medicine and Health Sciences, Faculty of Health Sciences, Linköping University, Department of Cardiology UHL, County Council of Östergötland, Linköping, Sweden

Tiny Jaarsma, PhD, RN, FAAN, FAHA, NFESC

Professor, Linkoping University

Department of Social- and Welfare Studies

Norrköping, Sweden

Anna Strömberg, PhD, RN, NFESC

Professor, Division of Nursing Science, Department of Medical and Health Sciences, Faculty of Health Sciences, Linköping University, Department of Cardiology UHL, County Council of

Östergötland, Linköping, Sweden

\author{
Corresponding Author \\ Dr. Barbara Riegel \\ Professor, School of Nursing \\ University of Pennsylvania \\ 418 Curie Boulevard \\ Philadelphia, PA 19104-4217 \\ 215-898-9927 (W) \\ 240-282-7707 (eFAX) \\ briegel@nursing.upenn.edu
}

Acknowledgements: The authors gratefully acknowledge the review of a prior draft of the manuscript provided by Afaf Meleis, PhD, RN, FAAN and by the doctoral students at the University of Pennsylvania. We also thank our faculty colleagues from Linkoping University who reviewed, critiqued, and discussed an early draft of this theory. 


\begin{abstract}
Nearly $50 \%$ of adults have one or more chronic illnesses. Self-care is considered essential in the management of chronic illness, but the elements of self-care in this context have not been specified in a middle-range theory. This article describes a middle-range theory of self-care that addresses the process of maintaining health with health promoting practices within the context of the management required of a chronic illness. The key concepts include self-care maintenance, self-care monitoring, and self-care management. Assumptions and propositions of the theory are specified. Factors influencing self-care including experience, skill, motivation, culture, confidence, habits, functional and cognition and support from others, and access to care are described.
\end{abstract}

Key words: chronic illness, middle-range theory, self-care 


\section{Introduction}

Middle range theories describe a view of reality that deals with specific phenomena and a limited number of variables. ${ }^{1}$ The $20^{\text {th }}$ century ended with considerable progress in the development of middle range theories in nursing. A recent search of the CINAHL (Cumulative Index to Nursing \& Allied Health) database revealed dozens of middle range theories addressing specific clinical phenomena with clear implications for nursing practice. Most of the middle range theories in the literature appear to have come directly from experience of nurse clinicians, although theories addressing pain, ${ }^{2}$ quality of life for the elderly, ${ }^{3}$ caregiver stress, ${ }^{4}$ and fatigue ${ }^{5}$ have evolved from the grand theories in nursing (eg, Roy, King, Neuman, Orlando).

Self-care is considered essential in the management of chronic illness, but the elements of self-care have not been specified in a middle-range theory that can be used across a variety of chronic conditions during the process of maintaining health. The aim of this article is to describe a middle-range theory of self-care in chronic illness, specifying the concepts, assumptions, propositions, and factors affecting self-care in this context. Intended and unintended outcomes of self-care are discussed and the manner in which the theory could illuminate and advance research and practice is described. This theory of self-care evolved from clinical practice caring for adults with heart failure. Those clinical experiences illustrated the challenges that patients had in caring for themselves when their decisions needed to be congruent with the needs dictated by their chronic condition. The theoretical base was formed from the situation-specific theory of heart failure self-care. ${ }^{6}$

While we acknowledge that health is a dynamic, subjective process that is experienced uniquely among individuals, for those who seek care within the organized health care system, chronic illness imposes a set of behavioral requirements. The majority of the patients seek care 
because they want relief from symptoms caused by their disease or its treatment. ${ }^{7}$ When providers interact with patients their intention is that the partnership they form will motivate patients to engage in self-care that can be incorporated into their lifestyle. It is within this context of a mutually rewarding relationship that the self-care of chronic illness takes place.

It would be disingenuous to ignore the influence of Dorthea Orem's grand theory on our work. The main difference between Orem's grand theory of self-care and this middle-range theory of self-care of chronic illness is that Orem's theory is not focused on chronic illness as is this middle range theory. Orem defined self-care as "the practice of activities that individuals initiate and perform on their own behalf in maintaining life, health and well-being" ${ }^{8}(\mathrm{p} .117)$. Our definition has similarities, but we address the process of self-care rather than specific activities. We focus primarily on individuals while Orem includes aspects of dependent care when others (self-care agents) initiate and perform activities to maintain life, health and well-being. In addition to the role of patients, Orem's theory evolved from her quest for greater understanding of the nature of nursing so she specifies how nurses promote self-care and assess self-care demands, abilities and deficits in order to support patients in their self-care. An assumption of her theory is that every patient should care for him/herself, but when an imbalance between selfcare needs and self-care abilities occurs there is a need for nursing intervention. Conversely, our goal was to codify the behaviors and processes used by individuals dealing with a diagnosis of a chronic illness and the attention given to nurses is limited. Further, rather than limiting this theory to nurses, we embrace all health care professionals as having a role in promoting selfcare. We believe that if health care professionals better understand the process used by patients in performing self-care, they can use this information to identify where patients struggle. This 
knowledge could be used to develop tailored interventions that improve outcomes for patients with chronic illness(es).

\section{Operational Definition of Concepts}

We define self-care as a process of maintaining health through health promoting practices and managing illness, as specified in our prior work. ${ }^{9}$ Self-care is performed in both healthy and ill states. Everyone engages in some level of self-care daily with tooth brushing, food choices, etc. When an ill individual is stable, s/he is often able to maintain health without moving into illness care. But, once one is ill and illness management becomes a priority, the need for selfcare for health remains. Thus, self-care in healthy and ill states can be, but is not always, a simultaneous process. However, self-care is relatively more salient to patients with a chronic illness and therefore we see a clear clinical need for a middle-range theory in this field. Engaging in self-care makes the patient an active participant in the management of illness. It should be noted that self-care is not a permanent, black or white phenomenon. In other words, self-care is not the same for all patients nor is it necessarily consistent over time. Indeed, some activities that might be defined as judicious self-care for one patient might be inappropriate for another.

Key concepts in this middle-range theory are self-care maintenance, self-care monitoring, and self-care management. Self-care maintenance is defined as those behaviors used by patients with a chronic illness to maintain physical and emotional stability. As described further below, these behaviors may be entirely self-determined or reflect recommendations that are mutually agreed on between patients and their health care provider. Self-care monitoring refers to the process of observing oneself for changes in signs and symptoms. Self-care management is 
defined as the response to signs and symptoms when they occur. Each of these concepts is discussed below. Note that these behaviors and activities will not always take place in the same, linear order and certain steps might be skipped.

Self-care maintenance. Self-care maintenance refers to those behaviors performed to improve well-being, preserve health, or to maintain physical and emotional stability. In well individuals self-care focuses on self-improvement, but in the face of a chronic illness, many selfcare maintenance behaviors mirror the recommendations of providers. These behaviors could be related to life-style (e.g. smoking cessation, preparing healthy food, coping with stress) or the medical regimen (e.g. taking medication as prescribed). These activities may be imposed by others (e.g. health care professionals or family members) and then agreed on by the patient or solely chosen by the patient to meet his/her own goals. Self-care maintenance benefits from reflection about the usefulness of the behavior, vigilance in performance of the behavior, and an on-going evaluation of benefits and the effectiveness of the activities. In addition, adaptation is often needed to accommodate changing conditions.

Adherence is an essential component of self-care maintenance. Adherence to therapies shown to be evidence-based is associated with the best outcomes. ${ }^{10}$ The goal for health care providers is to work collaboratively with patients to negotiate the adoption of as many of the advocated behaviors as the patient can tolerate and accept, with an emphasis on those therapies with the best evidence to support them.

Self-care monitoring. Self-care monitoring is a process of routine, vigilant body monitoring, surveillance, or "body listening". ${ }^{11}$ Monitoring is a normal human behavior. For example, many people monitor their weight regularly to follow gains and losses. Many visit the dentist to monitor their success in avoiding dental caries. In self-care of chronic illness, 
monitoring that is systematic and routine produces the best outcomes. For example, patients with asthma are encouraged to perform routine peak flow monitoring and symptom assessment and then intervene according to their asthma action plan to achieve the best possible health outcomes. ${ }^{12}$ Monitoring activities, such as checking blood sugar for diabetes, weighing daily for heart failure, and monitoring emotions in mental illness help to achieve physical and emotional stability. Three criteria are required for effective self-care monitoring. First, clinically significant changes over time in the condition must be possible. Second, a method of reliably detecting these changes must exist. Finally, a reasonable action must be possible in response.

The goal of self-care monitoring is recognition that a change has occurred. That change may be related to a chronic illness or not, but the self-care process under discussion here is the change occurring due to the chronic illness. Recognition of a physical change is facilitated by somatic awareness, defined as sensitivity to physical sensations and bodily activity. ${ }^{13}$ Recognition of an emotional change is aided by personal insight and interpersonal awareness. For individuals with chronic illness, sign or symptom recognition begins the process of decisionmaking about what action is needed. When signs and symptoms are detected early and the seriousness of them is understood, action can be taken before the situation escalates. Patients who are skilled in self-care monitoring can communicate information to a health care professional that will facilitate the provider's ability to give the best care. Self-care monitoring is the link between self-care maintenance and self-care management.

Self-care management. Self-care management involves an evaluation of changes in physical and emotional signs and symptoms to determine if action is needed. These changes may be due to illness, treatment, or the environment. Those who are best in self-care comprehend the meaning of changes and are able to mentally simulate options and decide on a course of action. If 
a response is needed, self-care management entails treatment implementation and the evaluation of the treatment. Treatments are often specific to the signs and symptoms of a particular chronic illness. For example, shortness of breath due to asthma may require use of a bronchodilator but shortness of breath due to heart failure may require taking an extra diuretic. Another important point about self-care management is that the treatment indicated may require consultation with a health care provider, depending on the messages the patient is given by the provider about independent modifications of therapies.

Finally, self-care management requires attention to the effectiveness of a treatment to evaluate whether or not that approach should be tried again in the future. Situation awareness promotes treatment evaluation by facilitating the perception of events, the comprehension of their meaning, and the projection of their status into the future. ${ }^{14}$ In this context, situation awareness involves alertness to bodily sensations (somatic awareness) and the ability to reliably determine how these sensations change in response to treatments implemented.

\section{Processes Underlying Self-Care}

Decision-Making. One underlying process that makes self-care so complex is the need for decision-making. In our early work, the management of symptoms was said to involve cognitive decision making undertaken in response to signs and symptoms. ${ }^{15}$ The basis of cognitive decision-making is classical decision theory, which prescribes analytical and systematic methods of weighing evidence prior to selecting a course of action. Later we came to realize that patients rarely use such a methodical, rational decision-making process. That is, they rarely generate and compare options in a systematic fashion. Naturalistic decision-making is a better description of the process used by patients in self-care. ${ }^{16}$ 
Naturalistic decision-making reflects the automatic, impulsive, contextual decisions that people make in complex real world situations. Real world situations are typically ambiguous, options are often vague, and decisions need to be made quickly. Real world decisions involve dynamically evolving conditions, uncertainty, ambiguity, missing information, time stress, and high stakes. These decisions may have goals that are ill-defined, shifting, or competing. Sometimes multiple individuals are involved in the decision making process. For example, patients with acute symptoms are facing a decision with important consequences. This situation will certainly evolve and the evolution may be rapid. A thorough decision-analysis is not a reasonable expectation in this situation.

Reflection. Reflection or contemplation is linked to knowledge acquisition, ${ }^{17}$ both of which are essential for self-care. As shown in figure 1, self-care may be sufficient or insufficient, purposive or unintentional, reasoned and reflective, or automatic and unobservant. This dichotomizing of the process builds on Orem's statement that "self-care is purposeful actions performed in sequence and with a pattern". 8 Among patients with low knowledge or poor indepth understanding of the rationale for self-care, many will perform little self-care of any quality (lower left quadrant). Perhaps more surprisingly, there will be those who perform selfcare activities but do so in an unreflective and unobservant fashion (top left quadrant). For example, many patients take their medications without knowing why they have been prescribed. Other patients dutifully monitor themselves, but do not know what to do with the information they gather. Presumably patients with inadequate knowledge could be involved in targeted education and thereby increase their self-care. However, the contribution of inadequate reflection needs to be addressed directly. Perhaps these patients would benefit from situation-based 
discussions that promote reflection. Research testing approaches to helping patients become reflective and purposive about their self-care is needed.

Other patients are reflective, but actively choose not to engage in self-care (bottom right quadrant). These individuals possess the knowledge and understanding of the self-care needed for their condition, but after reflection and perhaps a personal cost-benefit analysis, they make an informed choice not to perform the recommended self-care. This phenomenon is referred to in

the literature as reasoned decision-making, ${ }^{18}$ intelligent non-adherence, or self-regulation. ${ }^{19}$ Little has been written about the ethics of how to interact with chronically ill patients who choose not to perform self-care, but respect for autonomy and freedom of choice is essential. ${ }^{20}$ Motivational interviewing is one evidence-based approach to handling these challenging situations.

The ideal combination for a person with chronic illness is purposive, reflective, sufficient and reasoned self-care. Patients in this group (top right quadrant) are knowledgeable about the self-care needed for their condition. They perform self-care with thought about the meaning of the data they gather and make good decisions about what to do if signs or symptoms are abnormal. In order to reach a state of reflective, knowledgeable, sufficient self-care, patients are motivated and have received targeted education from a health care professional or have been very successful in seeking information and contemplating their options.

\section{Development of the Theory of Self-Care of Chronic Illness}

Building on our prior work and the conceptual work briefly outlined above, this middle range theory of self-care was developed in discussions among the authors regarding our prior work in self-care, ${ }^{21-23}$ the existing situation-specific theory of heart failure self-care, ${ }^{6}$ and the various self-care studies we have conducted with heart failure populations across the world. ${ }^{24-26}$ 
Discussions involved the meaning of the construct for a broader audience of patients with chronic illness and ways in which our prior, separate work could be integrated. First every concept and proposition of the situation-specific theory of heart failure self-care was evaluated and refined. A new version of the model was developed as a group and discussed as it applies to a variety of chronic illness states (Figure 2).

The major change made to the definitions of self-care maintenance and self-care management involved pulling out self-care monitoring as a separate concept. Here we specify that self-care monitoring is a distinct concept although it remains the bridge between self-care maintenance and self-care management with implications for both. In addition, an element of choice was added to self-care maintenance. That is, one may follow a low-fat diet, for example, because of the belief that it is required (i.e. imposed by another) or out of choice.

Finally, the literature on self-care is conceptually confusing. Numerous terms are used to refer to the same behavior (e.g. self-management, self-monitoring). In this theory we have clarified how various concepts can be integrated and used in relation to each other.

\section{Assumptions}

Premises accepted without proof are referred to as assumptions. This theory includes three assumptions:

1. There are differences between general self-care and illness-specific self-care. As noted in the introduction, in this article we are focusing on the self-care that occurs in the context of a chronic illness. General self-care is a dynamic, subjective process influenced by age, gender, culture, education, socioeconomic status, etc. Self-care that occurs in association with a chronic illness is circumscribed and influenced by others. 
2. Decision-making requires the ability to focus attention, to think, sufficient capacity for working memory and the ability to understand and weigh information.

3. Self-care for patients with multiple comorbid conditions may be conflicting when selfcare is considered for each illness separately. A growing body of literature illustrates the

complexity that patients face when trying to incorporate advice from multiple providers. ${ }^{27}$

\section{Propositions}

A scientific theory contains testable predictions or propositions. Seven propositions are proposed for this middle range theory of self-care.

1. There are core similarities in self-care across different chronic illnesses. For example, self-care monitoring is needed by patients with cancer, heart disease, diabetes, arthritis, and so forth.

2. Previous personal experience with illness or in caring for someone with a similar illness or with similar self-care needs increases the quality of self-care. That is, the processes of self-care are learned and that learning can occur through personal experience or during the process of caring for someone else.

3. Patients who engage in self-care that is purposive but unreflective are limited in their ability to master self-care in complex situations. Self-care is a learned process, so those who do not reflect on what they are learning have difficulty achieving a high level of mastery. Reflective self-care can be learned.

4. Misunderstandings, misconceptions, and lack of knowledge all contribute to insufficient self-care. This proposition captures the relationship between patients and the providers who devote their careers to helping patients master the knowledge needed for self-care. 
5. Mastery of self-care maintenance precedes mastery of self-care management because self-care maintenance is less complex than the decision-making required of self-care management.

6. Self-care monitoring for changes in signs or symptoms is necessary for effective self-care management because one cannot make a decision about a change unless it has been noticed and evaluated.

7. Individuals who perform evidence-based self-care have better outcomes than those who perform self-care that is not evidence-based.

\section{Intended and Unintended Outcomes of Self-Care}

One important intended outcome of self-care is illness stability, health, well-being and quality of life as implied in the term 'maintenance'. ${ }^{28}$ In addition to these, intended outcomes of self-care include an increase in perceived control over the illness and a decrease in the anxiety often associated with chronic illness. These patient-centered outcomes are of primary importance for this theory of self-care, but there is a growing body of literature suggesting that self-care can decrease hospitalization, cost and mortality, which has captured the attention of health economists. $^{29}$

Although the rapidity of treatment seeking behavior is a provider goal because of the known association with outcomes, rapid care seeking is not always the patients' goal, and unintended outcomes of self-care include a delay in treatment seeking. ${ }^{30}$ We demonstrated previously that maintaining a high level of perceived control during an acute illness event may increase treatment-seeking delay among patients with coronary artery disease. ${ }^{31}$ Others have found when an illness is acute, high perceived control plus low anxiety can cause self-deception, 
denial, and failure to seek care. ${ }^{32}$ Other unintended outcomes could include a higher burden associated with the need to perform self-care and guilt when one fails to follow treatment advice. ${ }^{33}$ In addition, self-care could actually increase illness costs by stimulating the patient to seek care even when it is not necessary.

\section{Factors Affecting Self-Care}

Self-care is an extremely challenging process, as evidenced by the burgeoning body of literature describing the challenges experienced by patients and clinicians alike. Some of the major factors serving as barriers and facilitators to self-care are described below.

Experience and Skill. Experience is one powerful contributor to the development of skill in self-care. Prior experiences are used to quickly identify patterns that provide relevant cues, suggest expected outcomes associated with specific responses, and point to reasonable goals and actions in specific types of situations. ${ }^{16}$ A simple pattern match may lead to recognition of the typicality of a situation, which would require a specific action..$^{34}$ Or, if the situation is unfamiliar, it might involve consideration of options evaluated by mental simulation.

Skill in self-care is essential and patients need to have the ability to plan, set goals, and make decisions. ${ }^{35,36}$ Experience also contributors to the development of skill, although some patients can have years of experience with a particular illness and never develop skill in selfcare. The challenge for health care professionals is to identify what patients have learned from experience, discern if what is known is correct, and facilitate the development of skills that are necessary for the performance of self-care.

Motivation. Motivation can be defined as the force driving humans to achieve their goals. Motivation is said to be intrinsic or extrinsic. Intrinsic motivation springs from the wish to 
assimilate and learn and includes aspect of enjoyment and interest. Intrinsic motivation is driven by an internal desire to perform a particular task because that task gives pleasure. On the other hand, extrinsic motivation refers to changing a behavior because it leads to a specific predetermined outcome that is desirable for some reason (e.g. to improve health, to please others). Many self-care behaviors are triggered and driven by extrinsic motivators, at least initially. That is, the individual may not be internally motivated to perform a behavior, but the perception of significant others regarding the importance of performing the behavior may motivate self-care. ${ }^{37}$

Cultural beliefs and values. Self-care might been seen as highly important in countries and cultures where independence is valued, but in some cultures self-care is not important. In these situations, it may be of greater importance to show love and concern through care and attention when a family member is ill. ${ }^{38}$ This behavior might also be relevant in cultures where collectivist ideals influence adoption of lifestyle changes. ${ }^{39}$ Sometimes self-care advice might contradict cultural beliefs, for example to promote rest in contrast to activity as recommended for patients with chronic heart failure. ${ }^{40}$

Confidence. Self-care is strongly influenced by attitudes and beliefs such as self-efficacy, defined as the confidence that one has in the ability to perform a specific action and to persist in performing that action despite barriers. ${ }^{41,42}$ The concept of confidence was discussed in our early work on self-care where confidence was conceptualized as a component of self-care. ${ }^{15}$ However, later testing revealed that confidence in the ability to perform self-care moderates the relationship between self-care and outcome ${ }^{43}$ leading us to conclude that confidence is not a part of self-care. ${ }^{6,44}$ Confidence in the ability to perform self-care is important in each stage of the self-care process. 
Habits. Habits or daily routines are important factors affecting self-care. Some patients get used to performing certain self-care behaviors and self-care becomes part of their daily routine. For other patients, however, self-care is considered 'work' ${ }^{33,45}$ For them, the regimen remains a continuous struggle. Perhaps those who are most successful in self-care are willing to adopt imposed behaviors until these behaviors evolve into habits with time.

Functional and Cognitive Abilities. Performing self-care requires the functional ability to engage in the required behaviors (e.g. balancing on a scale). Problems with hearing, vision, manual dexterity and energy can make self-care difficult. In addition, a growing body of knowledge illustrates that chronic illnesses are commonly associated with cognitive deficits that can make self-care particularly challenging. ${ }^{46}$

Support from Others. Although self-care is, by definition, performed by individuals, it would be naïve to suggest that self-care is always done alone. Instead, most chronically ill individuals would acknowledge the essential contributions (communication, decision making, and reciprocity) of family and friends - a process referred to as shared care when it involves two competent adults. $^{47}$

Access to care. Self-care of chronic illness is often influenced to some degree by providers after accessing the health care system to obtain care. However, it should be acknowledged that a large proportion of those with a chronic illness lack access to providers within the organized health care system for a variety of reasons (e.g. economic, location). These individuals obtain guidance from village elders, community workers, parents, neighbors, and friends. But, without access to trained health care providers, the outcomes associated with chronic illness are typically poor. ${ }^{48}$ 


\section{Clinical and Research Implications}

Not everyone is capable of performing self-care. Self-care is restricted to those who are able to make judicious decisions. ${ }^{49}$ Focused attention and working memory capacity are needed to perceive and process information prior to selecting a course of action and executing it.

Individuals with limited attention and memory have little ability to understand and reflect (e.g., infants, individuals with dementia) and thus may not be able to perform self-care. Those with severe psychiatric illness may have difficulty performing independent self-care. Even situational influences on attention and memory such as sleep deprivation or emotional stress can interfere

with self-care. ${ }^{50}$ In these situations shared care, dependent care, or community support is needed.

In conclusion, we believe that the theory proposed will be useful to both health care professionals and to researchers. In clinical practice, the theory can be used to structure an assessment of where in the self-care process a particular patient is struggling. Someone who is unable to recognize symptoms needs a very different intervention than someone who is poor in self-care maintenance. For example, symptom recognition may need skill while adoption of specific self-care maintenance behaviors may require motivation. Assessment of where in the process a particular patient is having difficulty will lead to a tailored intervention that may be more advantageous and cost-effective than a general intervention that addresses areas of self-care that the patient has already mastered. ${ }^{51}$

The usefulness of this theory to researchers is that the theory provides a structure that can focus research efforts and speed the attainment of results that can be translated into clinical practice. Specific suggestions for research include the development of measures of self-care for various chronic illnesses based on the theory. Intervention studies testing different types of approaches to improving self-care are greatly needed. Methods of promoting reflection published 
in the pedagogy literature could be adapted and tested to promote self-care. Culturally-relevant studies are needed to accommodate differences in particular cultural groups. Importantly, studies testing how self-care influences outcomes are greatly needed. 


\section{References}

1. Meleis Al. Theoretical Nursing: Development \& Progress. 4th ed. ed. Philadelphia: Lippincott williams \& Wilkins; 2007.

2. Good M. A middle-range theory of acute pain management: use in research. Nurs Outlook. MayJun 1998;46(3):120-124.

3. Register ME, Herman J. A middle range theory for generative quality of life for the elderly. ANS Adv Nurs Sci. Oct-Dec 2006;29(4):340-350.

4. Tsai PF. A middle-range theory of caregiver stress. Nurs Sci Q. Apr 2003;16(2):137-145.

5. Casalenuovo GA. Fatigue in diabetes mellitus: testing a middle range theory of well-being derived from Neuman's theory of optimal client system stability and the Neuman Systems Model: Nursing, University of Tennessee; 2002.

6. Riegel B, Dickson VV. A situation-specific theory of heart failure self-care. J Cardiovasc Nurs. May-Jun 2008;23(3):190-196.

7. Patel $\mathrm{H}$, Shafazand M, Schaufelberger M, Ekman I. Reasons for seeking acute care in chronic heart failure. Eur J Heart Fail. 2007;9(6-7):702-708.

8. Orem D. Nursing concepts of practice1991.

9. Riegel B, Carlson B, Moser DK, Sebern M, Hicks FD, Roland V. Psychometric testing of the selfcare of heart failure index. J Card Fail. Aug 2004;10(4):350-360.

10. Jaber WA, Lennon RJ, Mathew V, Holmes DR, Jr., Lerman A, Rihal CS. Application of evidencebased medical therapy is associated with improved outcomes after percutaneous coronary intervention and is a valid quality indicator. Journal of the American College of Cardiology. Oct 18 2005;46(8):1473-1478.

11. Dickson VV, Deatrick JA, Riegel B. A typology of heart failure self-care management in nonelders. Eur J Cardiovasc Nurs. Sep 2008;7(3):171-181.

12. Lung $\mathrm{CL}$, Lung ML. General principles of asthma management: symptom monitoring. The Nursing clinics of North America. Dec 2003;38(4):585-596.

13. Jurgens $\mathrm{CY}$. Somatic awareness, uncertainty, and delay in care-seeking in acute heart failure. Res Nurs Health. Apr 2006;29(2):74-86.

14. Endsley MR. The role of situation awareness in naturalistic decision making. In: Zsambok CE, Klein GA, eds. Naturalistic decision making: Lawrence Erlbaum Associates; 1996:436.

15. Riegel B, Carlson B, Glaser D. Development and testing of a clinical tool measuring selfmanagement of heart failure. Heart \& Lung. 2000;29(1):4-12.

16. Klein G. Naturalistic decision making. Hum Factors. Jun 2008;50(3):456-460.

17. Asselin ME. Using reflection strategies to link course knowledge to clinical practice: the RN-toBSN student experience. J Nurs Educ. Mar 2011;50(3):125-133.

18. Donovan JL, Blake DR. Patient non-compliance: deviance or reasoned decision-making? Soc Sci Med. Mar 1992;34(5):507-513.

19. Cameron LD, Leventhal H, eds. The self-regulation of health and illness behavior. London: Routledge; 2003.

20. McElmurry BJ, Zabrocki EC. Ethical concerns in caring for older women in the community. The Nursing clinics of North America. Dec 1989;24(4):1041-1050.

21. Jaarsma T, Stromberg A, Martensson J, Dracup K. Development and testing of the European Heart Failure Self-Care Behaviour Scale. European Journal of Heart Failure. 2003;5(3):363-370.

22. Jaarsma T, Abu Saad HH, Dracup K, Halfens R. Self-care behaviour of patients with heart failure. Scandinavian Journal of Caring Sciences. 2000;14(2):112-119. 
23. van der Wal MH, Jaarsma T, Moser DK, Veeger NJ, van Gilst WH, van Veldhuisen DJ. Compliance in heart failure patients: the importance of knowledge and beliefs. Eur Heart J. Feb 2006;27(4):434-440.

24. Holst M, Willenheimer R, Martensson J, Lindholm M, Stromberg A. Telephone follow-up of selfcare behaviour after a single session education of patients with heart failure in primary health care. Eur J Cardiovasc Nurs. Jun 2007;6(2):153-159.

25. Stromberg A, Martensson J, Fridlund B, Levin LA, Karlsson JE, Dahlstrom U. Nurse-led heart failure clinics improve survival and self-care behaviour in patients with heart failure: results from a prospective, randomised trial. Eur Heart J. Jun 2003;24(11):1014-1023.

26. Jaarsma $\mathrm{T}$, van der Wal MH, Lesman-Leegte I, et al. Effect of moderate or intensive disease management program on outcome in patients with heart failure: Coordinating Study Evaluating Outcomes of Advising and Counseling in Heart Failure (COACH). Arch Intern Med. Feb 11 2008;168(3):316-324.

27. Dickson VV, Buck H, Riegel B. A qualitative meta-analysis of heart failure self-care practices among individuals with multiple comorbid conditions. Journal of Cardiac Failure. May 2011;17(5):413-419.

28. Lucini D, Di Fede G, Parati G, Pagani M. Impact of chronic psychosocial stress on autonomic cardiovascular regulation in otherwise healthy subjects. Hypertension. Nov 2005;46(5):12011206.

29. Malmstrom RK, Roine RP, Heikkila A, et al. Cost analysis and health-related quality of life of home and self-care satellite haemodialysis. Nephrology, dialysis, transplantation : official publication of the European Dialysis and Transplant Association - European Renal Association. Jun 2008;23(6):1990-1996.

30. Gravely S, Tamim H, Smith J, Daly T, Grace SL. Non-symptom-related factors contributing to delay in seeking medical care by patients with heart failure: A narrative review. Journal of Cardiac Failure. 2011 publish ahead of print.

31. Riegel B, Elmi A, Moser DK, et al. Who listens to our advice? A secondary analysis of data from a clinical trial testing an intervention designed to decrease delay in seeking treatment for acute coronary syndrome. Patient education and counseling. Feb 52011.

32. Goldbeck R. Denial in physical illness. J Psychosom Res. Dec 1997;43(6):575-593.

33. Ekman I, Ehnfors M, Norberg A. The meaning of living with severe chronic heart failure as narrated by elderly people. Scand J Caring Sci. 2000;14(2):130-136.

34. Bond S, Cooper S. Modelling emergency decisions: recognition-primed decision making. The literature in relation to an ophthalmic critical incident. J Clin Nurs. Aug 2006;15(8):1023-1032.

35. Stromberg A. The crucial role of patient education in heart failure. Eur J Heart Fail. Mar 16 2005;7(3):363-369.

36. Dickson V, Riegel B. Are we teaching what patients need to know? Building skills in heart failure self-care. Heart \& Lung 2009;38:253-261.

37. Ajzen I, Fishbein M. The influence of attitudes on behavior. In: Albarracin D, Johnson BT, Zanna MP, eds. The Handbook of Attitudes: Lawrence Erlbaum Associates; 2005.

38. Greenhalgh T, Collard A, Campbell-Richards D, et al. Storylines of self-management: narratives of people with diabetes from a multiethnic inner city population. J Health Serv Res Policy. Jan 2011;16(1):37-43.

39. Galdas PM, Ratner PA, Oliffe JL. A narrative review of South Asian patients' experiences of cardiac rehabilitation. Journal of clinical nursing. Jul 62011.

40. Davidson PM, Macdonald P, Moser DK, et al. Cultural diversity in heart failure management: findings from the DISCOVER study (Part 2). Contemporary nurse : a journal for the Australian nursing profession. May-Jun 2007;25(1-2):50-61. 
41. Bandura A. Self-efficacy mechanism in human agency. American Psychologist. 1982;37:122-147.

42. van der Bijl J, Shortridge-Baggett $\mathrm{L}$. The theory and measurement of the self-efficacy construct. Scholarly Inquiry for Nursing Practice: An International Journal. 2001;15(3):189-207.

43. Kelly SJ, Stedman J, Leonardi-Bee J. Is hemoglobin A1c level associated with measures of socioeconomic status in non-diabetics after controlling for known explanatory factors. Stress and Health. 2005;21:185-192.

44. Riegel B, Lee CS, Dickson VV, Carlson B. An update on the self-care of heart failure index. J Cardiovasc Nurs. Nov-Dec 2009;24(6):485-497.

45. van der Wal MH, Jaarsma T, Moser DK, van Gilst WH, van Veldhuisen DJ. Qualitative examination of compliance in heart failure patients in The Netherlands. Heart Lung. Mar-Apr 2010;39(2):121-130.

46. Duff K, Mold JW, Roberts MM, McKay SL. Medical burden and cognition in older patients in primary care: selective deficits in attention. Archives of clinical neuropsychology : the official journal of the National Academy of Neuropsychologists. Jun 2007;22(5):569-575.

47. Sebern M. Shared Care, elder and family member skills used to manage burden. Journal of Advanced Nursing. Oct 2005;52(2):170-179.

48. Marra CA, Lynd LD, Esdaile JM, Kopec J, Anis AH. The impact of low family income on selfreported health outcomes in patients with rheumatoid arthritis within a publicly funded healthcare environment. Rheumatology. Nov 2004;43(11):1390-1397.

49. Naue U. 'Self-care without a self': Alzheimer's disease and the concept of personal responsibility for health. Medicine, health care, and philosophy. Sep 2008;11(3):315-324.

50. Lim J, Dinges DF. Sleep deprivation and vigilant attention. Ann N Y Acad Sci. 2008;1129:305-322.

51. Wangberg SC. An Internet-based diabetes self-care intervention tailored to self-efficacy. Health education research. Feb 2008;23(1):170-179. 


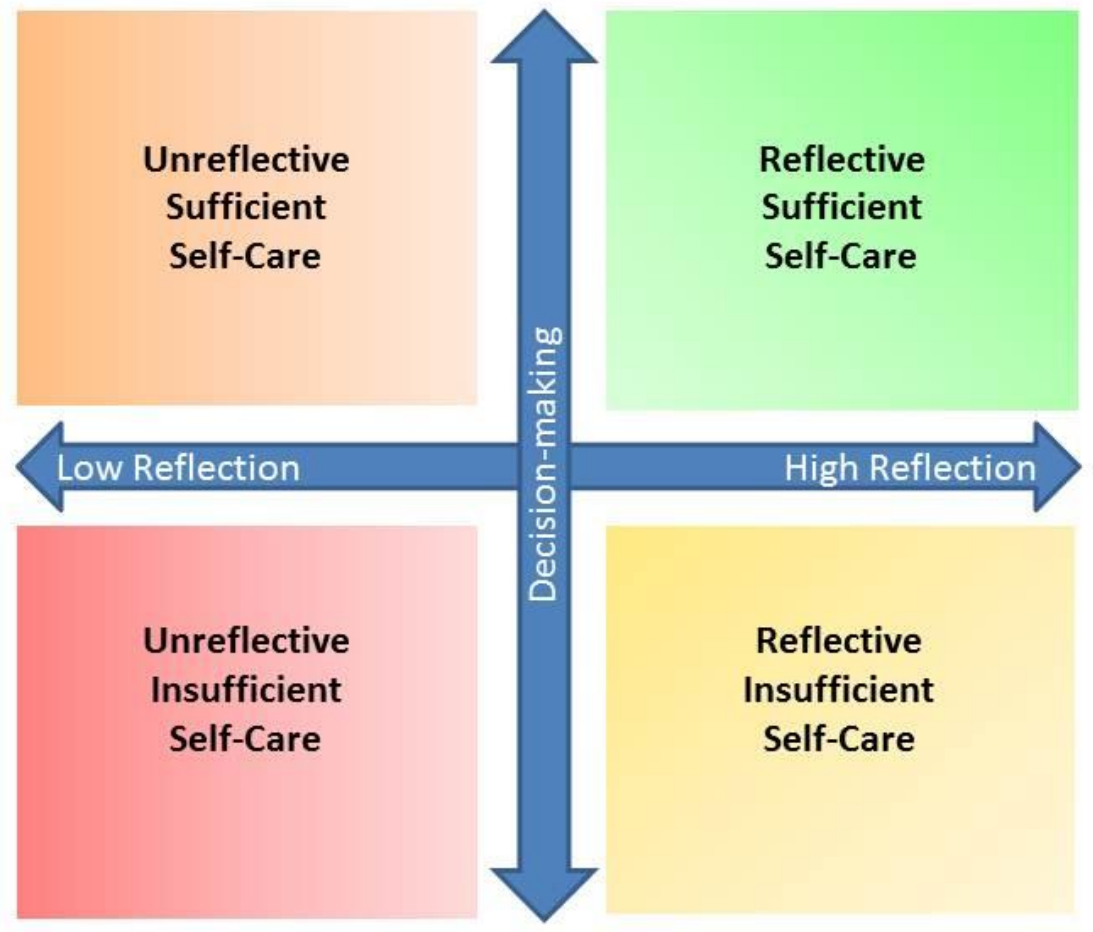

Figure 1: Relationship of decision-making and reflection on self-care. Self-care may be sufficient or insufficient, reasoned and reflective, or automatic and mindless. Various combinations of reflection and sufficiency of self-care are illustrated in this figure. The ideal combination is reflective and sufficient self-care. 


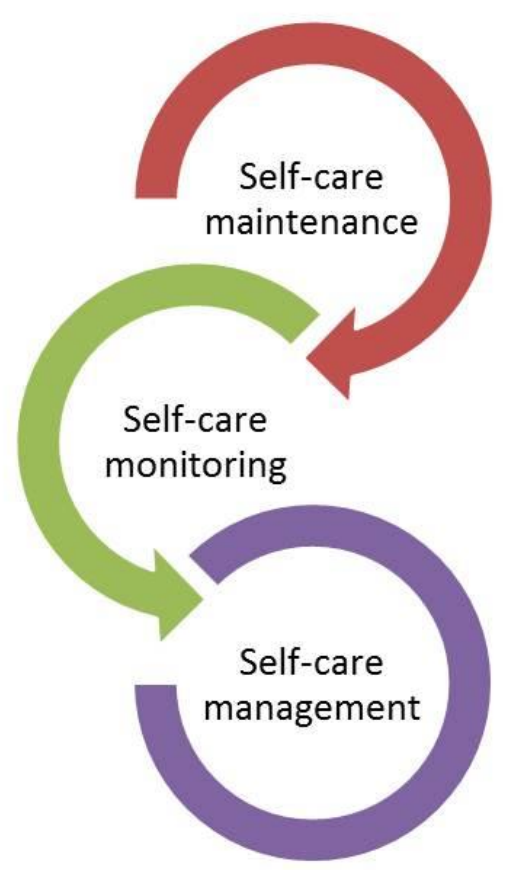

Figure 2: Self-care maintenance, self-care monitoring, and self-care management are core elements of the self-care of chronic illness. Self-care management entails a process of recognition of changes in signs and symptoms, decision-making about self-care actions, and evaluation of the outcomes of that action. Although each element is unique, they function in a synchronous fashion to maintain health and facilitate management of illness. As discussed further in the text, a variety of factors influence the success of individual patients in the performance of self-care. 